\title{
Processo de enfermagem informatizado para o cuidado a pacientes portadores de úlcera diabética: revisão integrativa da literatura
}

\author{
Computerized nursing process for the care of patients with diabetic ulcer: integrative \\ literature review
}
Proceso de enfermería informatizado para el cuidado de pacientes con úlcera diabética: revision integral de literatura

Ciro Rodrigo Rabelo da Mata1*, Nariani Souza Galvão², Sáskia Sampaio Cipriano de Menezes², Lisbeth Lima Hansen², Tracy Anne Ferreira de Oliveira Guimarães ${ }^{2}$, Aline Duarte Albuquerque ${ }^{2}$.

\section{RESUMO}

Objetivo: Analisar estudos que descrevam o desenvolvimento de softwares para a informatização do Processo de Enfermagem (PE) aplicado a indivíduos portadores de Úlcera Diabética (UD). Métodos: Revisão Integrativa da Literatura (RIL), nas bases de dados Literatura Latino-Americana e do Caribe em Ciências da Saúde (LILACS), Medical Literature Analysis and Retrieval System Online (MEDLINE), Índice Bibliográfico Espanhol em Ciências da Saúde (IBECS) e Base de Dados de Enfermagem (BDENF), com publicações em português, inglês e espanhol, no período de 2015 a 2019, disponibilizados na íntegra. Resultados: Foram avaliadas 236 publicações para elegibilidade, das quais selecionou-se 5 para análise. 4 artigos utilizaram a Teoria das Necessidades Humanas Básicas como referencial teórico. Predominou-se as taxonomias North-American Nursing Diagnosis Association (NANDA-I) e Nursing Interventions Classification (NIC). Apenas 1 informatizou as 5 etapas do PE e 3 estudos utilizaram o Sistema Android. Considerações finais: Identificou-se uma lacuna na literatura sobre o cuidado informatizado e sistematizado a essa população. Torna-se necessário o engajamento de universidades, instituições de saúde e fomento a pesquisas, no estímulo a iniciativas de ensino, pesquisa e extensão que evidenciem a importância da informática no apoio à tomada de decisão dos enfermeiros na assistência a indivíduos portadores de UD.

Palavras-chave: Processo de enfermagem, Pé diabético, Informática em enfermagem, Software.

\begin{abstract}
Objective: To analyze studies that describe the development of software for the computerization of the Nursing Process (NP) applied to individuals with Diabetic Ulcer (UD). Methods: Integrative Literature Review (ILR), in the databases Latin American and Caribbean Literature in Health Sciences (LILACS), Medical Literature Analysis and Retrieval System Online (MEDLINE), Spanish Bibliographic Index in Health Sciences (IBECS) and Base of Nursing Data (BDENF), with publications in Portuguese, English and Spanish, in the period from 2015 to 2019, available in full. Results: Were assessed 236 publications for eligibility, of which 5 were selected for analysis. 4 articles used the Theory of Basic Human Needs as a theoretical framework. Regarding the use of taxonomies, the North American Nursing Diagnosis Association (NANDA-I) and the Nursing Interventions Classification (NIC) predominated. Only 1 computerized the 5 stages of the NP and 3 studies used the Android System. Final considerations: A gap was identified in the literature regarding computerized and systematized care for this population. It is necessary to engage universities, health and promoting research institutions, to encourage teaching, research and extension initiatives, which highlight the importance of the informatics to support nurses' decision-making in assisting individuals with of DU.
\end{abstract}

Key words: Nursing process, Diabetic foot, Nursing informatics, Software.

\section{RESUMEN}

Objetivo: Analizar estudios que describen el desarrollo de softwares para la informatización del Proceso de Enfermería (PE) aplicado a individuos con Úlcera Diabética (UD). Métodos: Revisión Integral de Literatura (RIL), en las bases de datos Literatura Latinoamericana y del Caribe en Ciencias de la Salud (LILACS), Sistema de Análisis y Recuperación de Literatura Médica en línea (MEDLINE), Índice Bibliográfico Español

${ }^{1}$ Escola de Enfermagem de Manaus (EEM) - Universidade Federal do Amazonas (UFAM), Manaus-AM.

*E-mail: ciro-rodrigo@hotmail.com

SUBMETIDO EM: 7/2020 
en Ciencias de la Salud (IBECS) y Base de Datos de Enfermería (BDENF), con publicaciones en portugués, inglés y español, en el período de 2015 a 2019, en su totalidad. Resultados: Fueron seleccionadas para análisis 5 publicaciones. 4 artículos utilizaron la Teoría de las Necesidades Humanas básicas como marco teórico. Predominaron las taxonomías North-American Nursing Diagnosis Association (NANDA-I) y Nursing Interventions Classification (NIC). 1 computarizó las 5 etapas del PE y 3 estudios utilizaron el sistema Android. Consideraciones finales: Se identificó una brecha en la literatura con respecto a la atención computarizada y sistematizada para esta población. Es necesario que las universidades, instituciones de salud y de promoción de la investigación estimulen iniciativas de enseñanza, investigación y extensión, que resaltan la importancia de la informática para apoyar la toma de decisiones de las enfermeras en la atención a personas con UD.

Palabras clave: Proceso de enfermería, Pie diabético, Informática de enfermería, Software.

\section{INTRODUÇÃO}

A Úlcera Diabética (UD) é uma infecção que acomete tecidos moles dos membros inferiores, associada a alterações neurológicas e doença arterial periférica. Sua incidência é de 2 a $4 \%$, chegando a atingir uma prevalência de até $10 \%$ em países subdesenvolvidos e responsável por $85 \%$ das amputações (APELQVIST J, et al., 2000).

O Brasil é o quarto país com o maior número de pessoas com Diabetes Mellitus (DM) no mundo, com estimativa de 14,3 milhões de brasileiros que sofrem com a doença atualmente. Este cenário suscita medidas urgentes de controle e prevenção do DM e de suas complicações, caso contrário, o país estará fadado a ter um número expressivo de pessoas com sequelas graves e com expectativa de vida reduzida pelo alto índice de mortalidade (LUCOVEIS MLS, et al., 2018; IDF, 2013).

No movimento de modificar tal cenário, a enfermagem, por meio do Processo de Enfermagem (PE) como suporte à tomada de decisões, seja no âmbito gerencial ou assistencial, vem fornecer uma ferramenta que possibilita a identificação das demandas clínicas dos pacientes portadores de UD e assim responder às suas necessidades e em qualquer nível de atenção à saúde. O PE é definido como o instrumento metodológico e científico, implementado para viabilizar a prestação do cuidado qualificado ao indivíduo, à família ou à coletividade. Preconizado pela resolução 358/2009, é composto por cinco etapas interrelacionadas, interdependentes e recorrentes: Histórico de Enfermagem, Diagnóstico de Enfermagem, Planejamento de Enfermagem, Implementação e Avaliação de Enfermagem. Além disso, o Processo de Enfermagem deve ser direcionado por um referencial teórico que oriente a sua execução. Serve, ainda, como fundamentação para subsidiar atividades de educação, pesquisa e gerenciamento em Enfermagem (COFEN, 2009; LIMA JJ, et al., 2018).

Atualmente, as Tecnologias da Informação e Comunicação (TICs) têm sido utilizadas como um recurso para subsidiar o desenvolvimento do Processo de Enfermagem, pois permite integrá-lo em uma estrutura lógica de dados, informação e conhecimento para a tomada de decisão do cuidado de Enfermagem (DAL SASSO, et al., 2013). Dessa forma, utilizar-se dos avanços tecnológicos para planejar as atividades de assistência ao paciente de forma controlada e eficaz não se tornou apenas necessário, como também uma exigência crescente dos enfermeiros atualmente, pois sistemas de informação que retratem a realidade de sua prática (conhecimento tácito) e contenham elementos do conhecimento formal (explícito) facilitam a tomada de decisão clínica e documentação do cuidado, economizando tempo e custos, que podem ser redirecionados para a assistência. Ou seja, a inserção das TICs na prática assistencial preenche algumas lacunas que impossibilitam a utilização do Processo de Enfermagem na sua integralidade (SANTOS SR e NOBREGA MM, 2004).

Espera-se neste estudo o despertar de outros pesquisadores para a importância da iniciação tecnológica de enfermeiros e estudantes para o avanço técnico-científico da enfermagem, apontando para os avanços até aqui publicados e indicando lacunas no conhecimento sobre o tema pesquisado. $O$ objetivo da presente revisão foi analisar estudos que descrevam o desenvolvimento de softwares voltados para a informatização do PE aplicado ao cuidado de indivíduos portadores de UD. 


\section{MÉTODOS}

Trata-se de uma revisão integrativa de Literatura (RIL), cujo protocolo seguiu os seguintes passos metodológicos: elaboração da pergunta de pesquisa; seleção dos descritores; definição dos critérios de seleção; levantamento do material bibliográfico; organização das categorias e análise dos dados obtidos (SOARES CB, et al., 2014; SOUZA NR, et al., 2017). A elaboração da questão de pesquisa foi constituída por meio da estratégia PICO, segundo Barbosa RFM, et al. (2019) e Melnyk BM e Fineout-overholt E (2005): P - população do estudo e/ou situação-problema (estudos que descrevem o desenvolvimento de softwares voltados para a informatização do PE aplicado a indivíduos portares de UD); I- intervenção estudada ou variável de interesse (PE para tomada de decisão mediante informatização); C- comparação com outra intervenção (PE para tomada de decisão sem ajuda da informatização); O- desfecho (a integração de dados pela informatização facilita a tomada de decisão). Assim, a revisão se pautou na seguinte questão norteadora: "O que tem sido publicado/produzido sobre a construção/elaboração de softwares direcionados para o cuidado de enfermagem sistematizado ao portador de úlcera diabética?"

Como estratégia de busca, foram consultadas as bases de dados: Medical Literature Analysis and Retrieval System Online (MEDLINE), Literatura Latino-Americana em Ciências da Saúde (LILACS), Índice Bibliográfico Espanhol em Ciências da Saúde (IBECS) e Base de Dados de Enfermagem (BDENF), primeiramente com os descritores "Processo de Enfermagem"/“Nursing Process", "Software" e "Pé Diabético"/"Diabétic Foot", isoladamente e combinados entre si, com os operadores booleanos AND e OR. Os critérios de inclusão adotados para a busca e seleção das publicações foram: artigos publicados em periódicos científicos nacionais e internacionais que abordassem a temática do PE informatizado, publicados em língua portuguesa, inglesa ou espanhola, no período de 2015 a 2019 e indexados nas bases de dados: MEDLINE, LILACS, IBECS e BDENF, disponibilizados na íntegra, diretamente pelo site da base ou pelo Portal Capes. A estratégia de busca dos estudos nas bases de dados está descrita na Figura 1.

Figura 1- Fluxograma de busca e seleção dos estudos nas bases de dados. Manaus-AM, Brasil, 2020.

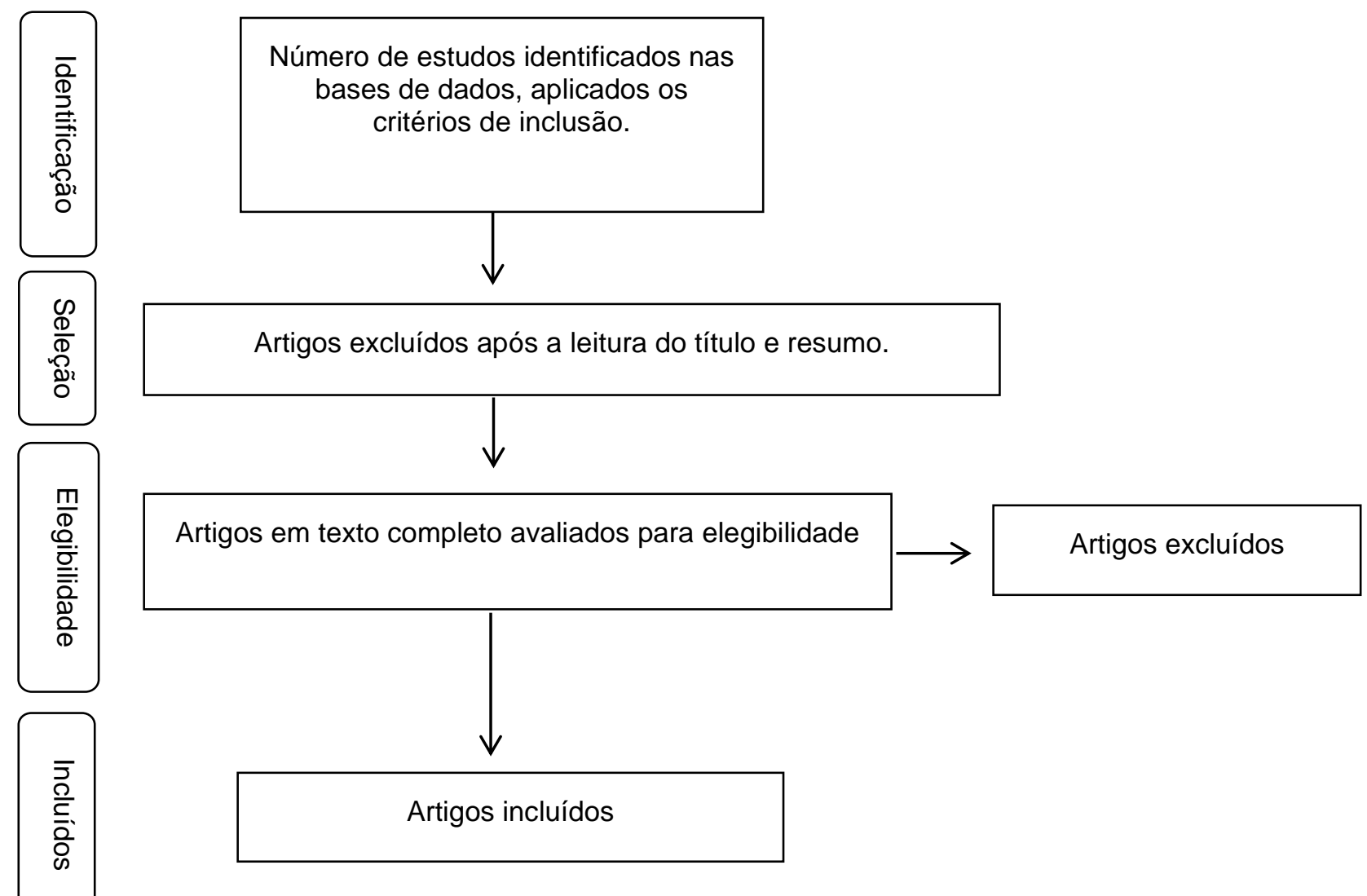

Fonte: Mata CRR, et al., 2020. 


\section{RESULTADOS}

Partindo da pergunta de revisão, durante a pesquisa nas bases a partir das estratégias de busca, não foram encontrados estudos que descrevessem a informatização do PE, mas sim tecnologias voltadas para diagnóstico e tratamento de feridas crônicas em geral, fora do campo de atuação específica do enfermeiro. Posteriormente, foram utilizados isoladamente e combinados com os operadores booleanos AND e OR, nas bases de dados LILACS, MEDLINE, IBECS e BDENF, os descritores "Processo de Enfermagem", "Software", "Ferimentos e Lesões" e "Cuidados de Enfermagem", cadastrados no Portal de Descritores das Ciências da Saúde (DeCS). Na base Medical Literature Analysis and Retrieval System Online, foi realizada investigação a partir dos descritores da terminologia Medical Subject Headings (MeSH), isolados e combinados pelos operadores booleanos AND e OR: "Nursing Process", "Software", "Wounds and Injuries" e "Nursing Care".

Nessa tentativa, foram encontrados artigos que descreviam as etapas de informatização do PE, mas não contemplavam a população em estudo: portadores de feridas crônicas/úlcera diabética. Por fim, devido a relevância técnico-científica dos artigos encontrados e a lacuna na literatura acerca de estudos que direcionem o cuidado de enfermagem sistematizado e informatizado aos portadores de feridas crônicas, nas referidas bases, decidiu-se por analisar os artigos que descrevessem o processo de informatização do PE em outros contextos assistenciais, e também que evidenciassem os requisitos que um software na área de enfermagem precisaria ter para orientar a sistematização da assistência. Dessa forma, a busca e seleção dos estudos incluídos na presente revisão está descrita na Figura 2.

Figura 2 - Fluxograma de busca e seleção dos estudos incluídos na revisão. Manaus-AM, Brasil, 2020.

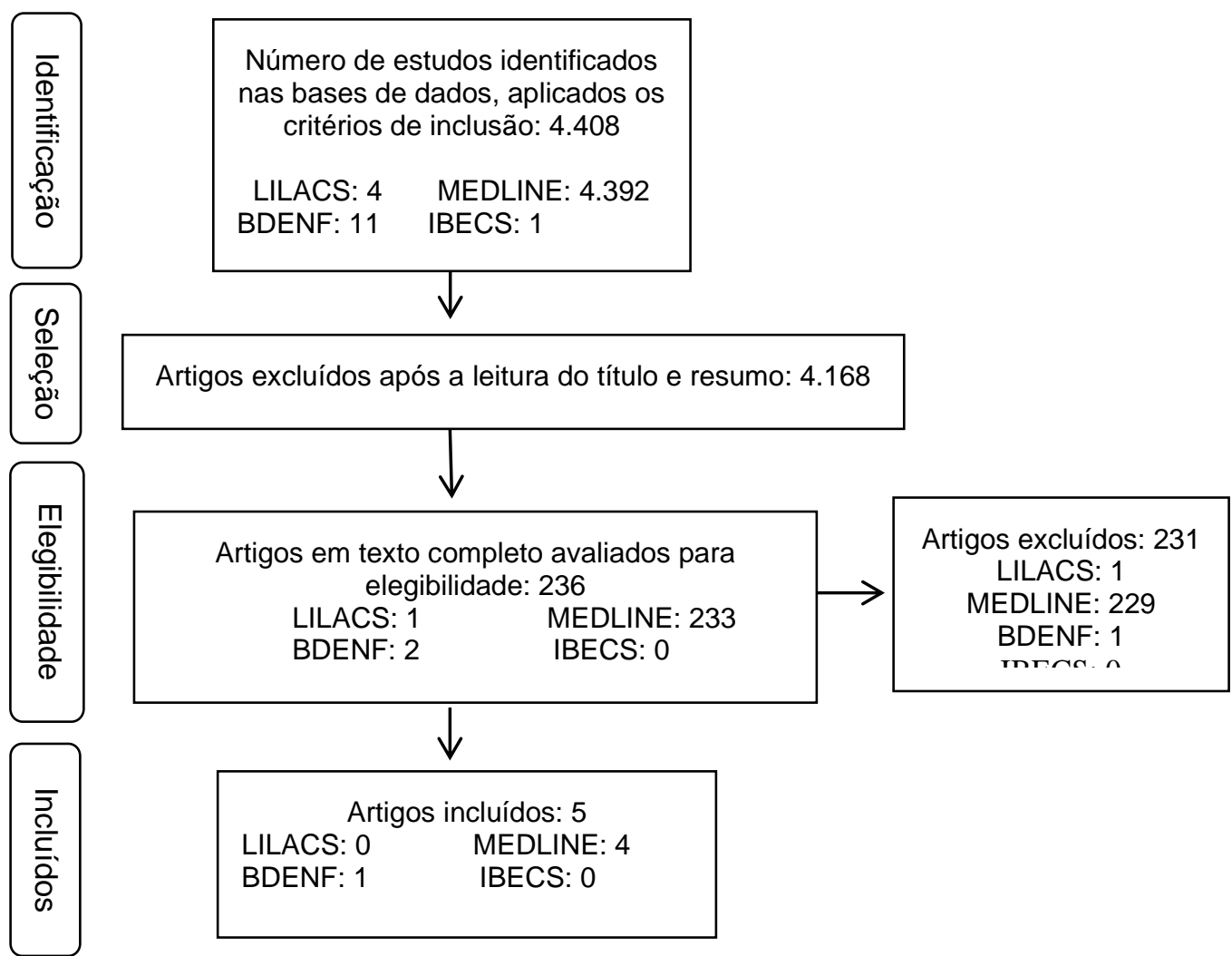

Fonte: Mata CRR, et al., 2020.

O Quadro 1 apresenta a descrição dos estudos selecionados para esta revisão, descrevendo título, autoria, periódico, ano de publicação, país de publicação, objetivos, delineamento metodológico e base de dados de origem. É possível observar, quanto a origem das publicações, que os 5 artigos selecionados são brasileiros, sendo 1 da região Norte, 3 da região Nordeste e 1 da região Sudeste. 
Quadro 1- Descrição dos estudos selecionados quanto ao: título, autores/periódico/ano e país de publicação, objetivos e delineamento metodológico. Manaus-AM, Brasil, 2020.

\begin{tabular}{|c|c|c|c|c|}
\hline $\begin{array}{c}\text { Autores/Periódico/Ano } \\
\text { de publicação }\end{array}$ & $\begin{array}{l}\text { País da } \\
\text { Publicação }\end{array}$ & Objetivos & $\begin{array}{l}\text { Delineamento } \\
\text { Metodológico }\end{array}$ & $\begin{array}{l}\text { Base de } \\
\text { dados }\end{array}$ \\
\hline $\begin{array}{l}\text { Junior MGS, et al./ Rev } \\
\text { Bras Enferm/2018 }\end{array}$ & Brasil & $\begin{array}{l}\text { Descrever } \\
\text { desenvolvimento de um } \\
\text { software protótipo para } \\
\text { aplicar o Processo de } \\
\text { Enfermagem em unidades } \\
\text { de clínica médica de um } \\
\text { hospital geral e avaliar sua } \\
\text { utilidade. }\end{array}$ & $\begin{array}{l}\text { Pesquisa } \\
\text { metodológica } \\
\text { aplicada de } \\
\text { produção } \\
\text { tecnológica }\end{array}$ & MEDLINE \\
\hline $\begin{array}{l}\text { Lima JJ, et al./ Rev Bras } \\
\text { Enferm/2017 }\end{array}$ & Brasil & $\begin{array}{l}\text { Construir uma tecnologia } \\
\text { móvel para auxiliar o } \\
\text { enfermeiro na coleta de } \\
\text { dados, no raciocínio } \\
\text { diagnóstico } \\
\text { identificação de possíveis } \\
\text { intervenções em neonatos. }\end{array}$ & $\begin{array}{l}\text { Pesquisa } \\
\text { metodológica } \\
\text { e de } \\
\text { desenvolvimen } \\
\text { to tecnológico }\end{array}$ & MEDLINE \\
\hline $\begin{array}{l}\text { Melo ECA, et al./ Rev } \\
\text { Bras Enferm/2017 }\end{array}$ & Brasil & $\begin{array}{l}\text { Descrever as etapas de } \\
\text { construção e transição } \\
\text { realizadas no } \\
\text { desenvolvimento de um } \\
\text { ambiente virtual de } \\
\text { aprendizagem (Plataforma } \\
\text { PEnsinar®) voltado para o } \\
\text { ensino do processo de } \\
\text { enfermagem e das classifi } \\
\text { cações Nanda } \\
\text { Internacional, NIC, NOC e } \\
\text { CIPE®. }\end{array}$ & $\begin{array}{l}\text { Pesquisa } \\
\text { metodológica } \\
\text { e de } \\
\text { desenvolvimen } \\
\text { to tecnológico }\end{array}$ & MEDLINE \\
\hline $\begin{array}{l}\text { Silva KL, et al./ Rev } \\
\text { Latino-Am } \\
\text { Enfermagem/2015 }\end{array}$ & Brasil & $\begin{array}{l}\text { Relatar o desenvolvimento } \\
\text { de um software de apoio à } \\
\text { tomada de decisão na } \\
\text { seleção de diagnósticos e } \\
\text { intervenções de } \\
\text { Enfermagem para crianças } \\
\text { e adolescentes, a partir da } \\
\text { nomenclatura de } \\
\text { diagnósticos, resultados e } \\
\text { intervenções de } \\
\text { Enfermagem de um } \\
\text { hospital universitário da } \\
\text { Paraíba. }\end{array}$ & $\begin{array}{l}\text { Pesquisa } \\
\text { metodológica } \\
\text { e de } \\
\text { desenvolvimen } \\
\text { to tecnológico }\end{array}$ & MEDLINE \\
\hline $\begin{array}{l}\text { Miranda LN, et al./Rev } \\
\text { Enferm UFPE } \\
\text { Online/2017 }\end{array}$ & Brasil & $\begin{array}{l}\text { Analisar as especificações } \\
\text { de sistemas similares no } \\
\text { intuito de descobrir que } \\
\text { requisitos deve conter um } \\
\text { software para à assistência } \\
\text { de Enfermagem. }\end{array}$ & $\begin{array}{l}\text { Revisão } \\
\text { Integrativa de } \\
\text { Literatura }\end{array}$ & BDENF \\
\hline
\end{tabular}

Fonte: Mata CRR, et al., 2020. 
Em relação à distribuição desses estudos por ano de publicação, em 2017 foram identificadas 3 publicações, nos anos de 2015 e 2018, apenas 1 publicação em cada ano. A revista que mais publicou sobre a temática foi a Revista Brasileira de Enfermagem (REBEN), com 3 publicações, seguida pela Revista Latino-Americana de Enfermagem (RLAME) e Revista de Enfermagem UFPE On Line (JNUOL), com 1 publicação cada.

Quanto ao delineamento metodológico, evidenciou-se que 4 estudos são de natureza metodológica e de desenvolvimento tecnológico, que buscam descrever o processo de desenvolvimento de softwares para informatizar o PE (LIMA JJ, et al., 2018; SILVA KL, et al., 2015; MELO ECA, et al., 2018; JUNIOR MGS, et al., 2018).

3 estudos propuseram softwares para o contexto assistencial (LIMA JJ, et al., 2018; SILVA KL, et al., 2015; JUNIOR MGS, et al., 2018) enquanto 1 descreveu o desenvolvimento de um software educacional para o ensino do Processo de Enfermagem, utilizando a abordagem teórica sócio-histórica de Lev Vygotsky, pesquisador russo, com destaque na área de aprendizagem e desenvolvimento (MELO ECA, et al., 2018).

Dentre os 5 estudos selecionados para análise, apenas um classifica-se como uma RIL, cujo objetivo foi analisar as especificações de sistemas similares no intuito de descobrir que requisitos são necessários ao desenvolvimento de um software para a assistência de enfermagem. Mesmo discordando do padrão de estudos metodológicos e de desenvolvimento tecnológico, as discussões e resultados apresentados pelo artigo são de extrema relevância para análise do tema proposto, pois apresenta evidências na literatura sobre os requisitos para um software e assim apoiar a execução do PE na prática clínica dos enfermeiros (MIRANDA LN, et al., 2017).

Em relação ao referencial teórico escolhido para orientar a execução do PE informatizado, 4 artigos utilizaram a Teoria das Necessidades Humanas Básicas de Wanda Horta, principalmente por se tratar de uma teoria já utilizada para nortear o cuidado nos contextos assistenciais aos quais foram propostos a informatização do PE (LIMA JJ, et al., 2018; SILVA KL, et al., 2015; MELO ECA, et al., 2018; JUNIOR MGS, et al., 2018).

Quanto à informatização das cinco etapas do processo de enfermagem, apenas em 2 estudos (MELO ECA, et al., 2018; JUNIOR MGS, et al., 2018) todas as etapas foram contempladas. Nenhum dos demais estudos utilizou a Nursing Outcomes Classification (NOC) na terceira etapa, conhecida como Planejamento de Enfermagem.

Referente à escolha dos sistemas de classificações de enfermagem, houve predominância das taxonomias North-American Nursing Diagnosis Association (NANDA-I) e Nursing Interventions Classification (NIC). No estudo de Melo ECA, et al. (2018), entretanto, havia a funcionalidade de o usuário optar pelo "Módulo NANDA-NOC-NIC" ou "Módulo CIPE".

De acordo com a Quadro 2, evidenciou-se que houve certa diversidade de ferramentas de programação utilizadas; 3 estudos utilizaram o Sistema Android pelas vantagens de mobilidade, manuseio, capacidade de armazenamento e facilidade de acesso pelo toque dos dados (LIMA JJ, et al., 2018; MELO ECA, et al., 2018; JUNIOR MGS, et al., 2018).

Apenas o desenvolvido por Junior MGS, et al. (2018) validou o aplicativo, pois norteou-se pela engenharia do software com base no modelo de prototipação, que incluía a fase de validação, obtendo êxito em seu objetivo de apoiar o desenvolvimento do PE na clínica médica de um hospital no Pará. Houve predominância na escolha pela Engenharia de Software proposta por Pressman RS e Maxim BR (2016), como método de desenvolvimento, o qual é dividido em três fases: Planejamento, Modelagem e Construção (LIMA JJ, et al., 2018). 
Quadro 2 - Métodos de programação dos softwares nos estudos selecionados. Manaus-AM, Brasil, 2019.

\begin{tabular}{|c|c|}
\hline $\begin{array}{l}\text { Título do Estudo } \\
\end{array}$ & Método de programação do software \\
\hline $\begin{array}{l}\text { Desenvolvimento de software para apoiar a } \\
\text { tomada de decisão na seleção de diagnósticos e } \\
\text { intervenções de enfermagem para crianças e } \\
\text { adolescentes }\end{array}$ & $\begin{array}{l}\text { Engenharia de software proposto por Pressman e } \\
\text { fundamentado no ciclo de vida de desenvolvimento } \\
\text { de sistemas; Linguagem Unificada de Modelagem } \\
\text { (UML-Unified Modeling Language); fluxograma } \\
\text { elaborado com auxílio do JUDE System Design } \\
\text { Tool,; utilizou-se o software Axure RP® para } \\
\text { desenvolver a interface de navegação das telas e } \\
\text { facilitar a visualização do Processo de } \\
\text { Enfermagem. }\end{array}$ \\
\hline $\begin{array}{llll}\text { Plataforma } & \text { PEnsinar } \AA \text { : } & \text { ferramenta } & \text { de } \\
\text { aprendizagem } & \text { para } 0 \text { ensino do processo } & \text { de } \\
\text { enfermagem } & & & \end{array}$ & $\begin{array}{l}\text { Metodologia baseada na engenharia de software, } \\
\text { chamada de Rational Unified Process (RUP). Esse } \\
\text { processo é constituído pelas etapas de concepção, } \\
\text { elaboração, construção, transição e produção. } \\
\text { Neste estudo foram apresentadas apenas as } \\
\text { etapas de construção e transição. }\end{array}$ \\
\hline $\begin{array}{l}\text { Processo de enfermagem } \\
\text { construção de tecnologia móvel para uso em } \\
\text { neonatos }\end{array}$ & $\begin{array}{l}\text { Modelo cascata ou ciclo de vida clássico, proposto } \\
\text { por Winston Royce, com base no modelo proposto } \\
\text { por Pressman \& Maxim (2016); Uso das linguagens } \\
\text { de desenvolvimento RubyonRails, IONIC 2, } \\
\text { PostgresSQL e Amazon. }\end{array}$ \\
\hline $\begin{array}{l}\text { Software para Sistematização da Assistência de } \\
\text { Enfermagem em unidade de internação hospitalar }\end{array}$ & $\begin{array}{l}\text { Engenharia do software com base no modelo de } \\
\text { prototipação, desenvolvido em três fases: } \\
\text { especificação, desenvolvimento e validação. } \\
\text { Foram utilizadas as tecnologias: Python - como } \\
\text { linguagem de programação, Django - como } \\
\text { webframework construído com a linguagem } \\
\text { Python,; Postgresq1. }\end{array}$ \\
\hline $\begin{array}{l}\text { Sistema de tomada de c } \\
\text { revisão integrativa }\end{array}$ & Revisão Integrativa de Literatura; não se aplica. \\
\hline
\end{tabular}

Fonte: Mata CRR, et al., 2020.

\section{DISCUSSÃO}

\section{Benefícios advindos com a inserção da informática à prática de enfermagem}

A utilização de recursos tecnológicos traz uma série de vantagens e melhorias na atuação do enfermeiro, pois além aumentar o tempo disponível para as atividades assistenciais, pode impulsionar a pesquisa, o ensino e a práxis na enfermagem; auxiliar a documentação das ações de cuidado e também ajudar tanto profissionais quanto estudantes no desenvolvimento do julgamento clínico e do processo de raciocínio diagnóstico. Podem contribuir, ainda, para se avaliar a acurácia do diagnóstico e elevar a qualidade do cuidado prestado (SOARES CB, et al., 2014; SILVA KL, et al., 2015).

Dessa forma, reconhecendo que as ferramentas computacionais são capazes de agregar tantas vantagens para a prática clínica, verifica-se a necessidade de buscar nas TICs uma nova forma de executar e registrar o PE, a fim de proporcionar um registro informatizado, individualizado, rápido e eficiente para cada cliente (GARCIA TR e NÓBREGA MML, 2009). Essa informação corrobora com os resultados de um estudo incluído na presente revisão, autoria de Domingos CS, et al. (2017), o qual constatou a facilidade para fazer as prescrições de enfermagem com rapidez e segurança, de maneira digital, quando comparada à prescrição manual.

Os estudos analisados nesta revisão salientam a importância dos sistemas de informação na área da enfermagem como parte integrante da equipe de saúde, oferecendo visibilidade à sua atuação, apoiando a tomada de decisão. Oportuno ressaltar que o uso das TICs não visa a substituição do pensamento crítico e raciocínio clínico do enfermeiro, fato que exige dos profissionais constante atualização de conhecimentos técnico-científicos que subsidiem o PE (JUNIOR MGS, et al., 2018). 


\section{Dificuldades na construção e implementação de softwares voltados para informatização do PE}

Ainda que se apresentem como excelente alternativa para a melhoria da qualidade do cuidado nos respectivos contextos assistenciais, os estudos de desenvolvimento tecnológico incluídos na presente revisão apresentaram as seguintes limitações: necessidade de realização de testes de usabilidade e da qualidade em uso, uma vez que os testes com o sistema foram realizados em ambientes simulados (SILVA $\mathrm{KL}$, et al., 2015); dificuldade de acessar pesquisadores e discentes da área tecnológica que tenham interesse por projetos orientados à saúde (MELO ECA, et al., 2018); limitações quanto ao uso de escalas de avaliação dos cuidados prescritos, por meio da taxonomia NOC, devido ao reduzido espaço para incorporação de telas com esta finalidade; o elevado custo financeiro para a construção do software (LIMA $\mathrm{JJ}$, et al., 2018); e a não continuidade das ações implementadas no cotidiano das unidades de assistência à saúde onde se realizou o estudo (JUNIOR MGS, et al., 2018).

Ainda que inovador, o uso das TICS para apoiar a execução do PE pode ser algo desafiador, pois, no cenário da enfermagem, percebe-se certa resistência de alguns profissionais à apropriação e posterior utilização das tecnologias como ferramentas para integração, nas várias dimensões do cuidado. Tal fato, é constatado no estudo de Junior MGS, et al. (2018), incluído nesta revisão, o qual afirma que pode comprometer, portanto, todos os benefícios já evidenciados, uma vez que um dos principais objetivos da inserção das TICS na área de enfermagem é apoiar a prática clínica dos enfermeiros.

Além disso, mesmo vencidos os impasses em relação a adesão das equipes, a informatização do PE pode não acontecer de maneira equânime e na mesma velocidade no Brasil. Dentre os estudos selecionados, duas das iniciativas exitosas se concentraram na região Nordeste, e em revisão recente sobre o assunto realizada por Pissaia LF, et al. (2018), dos 15 estudos analisados, 9 eram da região Sudeste, apontando para uma disparidade, em termos de produção científica, entre as regiões brasileiras referente ao desenvolvimento do registro eletrônico de enfermagem (SILVA KL, et al., 2015). O único artigo de revisão incluído neste estudo Miranda LN, et al. (2017), o qual analisa os requisitos de um software para a execução do PE, identificou fragilidades nos artigos incluídos. Dos 23 estudos selecionados, nenhum apoiava o desenvolvimento de todas as etapas do PE, e apenas 4 softwares indicavam 4 das 5 das etapas do PE, sendo elas: coleta de dados, diagnóstico de enfermagem, implementação e avaliação.

Na presente revisão, dos 4 artigos de desenvolvimento tecnológico incluídos, apenas dois (MELO ECA, et al., 2018; JUNIOR MGS, et al., 2018) apoiavam o desenvolvimento de todas as etapas do PE. Nos outros três (LIMA JJ, et al., 2017; SILVA KL, et al., 2015; MIRANDA LN, et al., 2017), identificou-se que o principal entrave está na $3^{\underline{a}}$ etapa, o planejamento de enfermagem, cuja taxonomia empregada é a NOC. Esse cenário aponta para a necessidade de futuras pesquisas no âmbito da informatização do PE e ao estudo da aplicabilidade clínica da NOC, uma vez que que a execução do PE em sua integralidade é condição indispensável à promoção do cuidado integral e seguro aos pacientes.

\section{Barreiras ao desenvolvimento de softwares voltados para o cuidado de Enfermagem especializado ao portador de UD}

Em estudo de revisão realizado por Camargo FC, et al. (2018), foram analisados artigos nacionais relacionados ao uso de softwares para o apoio ao raciocínio clínico dos enfermeiros, evidenciou-se que apesar da ampla busca por estudos primários, identificou-se uma incipiente produção sobre o tema. Em média 0,6 publicações/ano para o período de 1997 a 2017.

Outro ponto crítico é que o ensino do PE, abrangendo as classificações, constitui-se como um desafio para a formação do profissional nas escolas de enfermagem (PISSAIA LF, et al., 2018), isso se deve às lacunas na produção científica sobre o ensino deste método no Brasil, e pela ausência de políticas institucionais que facultem a integração entre ensino, pesquisa e assistência. Para assegurar o ensino do PE de modo transversal, convém que as Instituições de Ensino Superior (IES) promovam contínua qualificação e capacitação pedagógica, além de viabilizar políticas de educação permanente nos serviços. Oportunamente a participação ativa de enfermeiros em diversas instâncias e órgãos representativos, busquem refletir e debater sobre os aspectos teórico-filosóficos do PE nas searas do ensino e assistência. 
Tal dificuldade de associação teórico-prática pode ser atribuída ao que é ensinado nas universidades, que muitas vezes não corresponde à realidade vivenciada no SUS. Recente revisão de literatura (SILVA IAS, et al., 2018) sobre o ensino do PE no Brasil constatou que os hospitais privados apresentam maior percentual de implementação da SAE, quando comparados aos da rede pública. O contexto descrito pode estar relacionado com o processo de trabalho da enfermagem no âmbito do SUS, o conhecimento limitado, por parte dos profissionais de enfermagem, sobre do $\mathrm{PE}$, número insuficiente de instrumentos de coleta de dados e documentação do cuidado, dimensionamento de pessoal inadequado às demandas assistenciais $\mathrm{e}$ infraestrutura insalubre (CARVALHO EC, et al., 2007).

Todo esse panorama colabora com a fragilidade do ensino e pesquisa em enfermagem no Brasil, fato que implica diretamente no incentivo e qualidade da informatização do PE, alertando para a necessidade de fomento e apoio institucional das entidades brasileiras de saúde e de educação em enfermagem no sentido de viabilizarem produções científicas nessa área, contribuindo com a valorização do ensino e implementação do PE por meio da integração pesquisa, ensino e assistência. Faz-se necessário salientar, entretanto, que não se pode atentar apenas para a problemática da quantidade insuficiente de estudos e da fragilidade do ensino do $\mathrm{PE}$, no Brasil. É preciso também avaliar a qualidade da inserção dessas tecnologias na assistência em consonância com os modelos de atenção à saúde no Brasil. Esse fato pode ser evidenciado por recente revisão feita por Domingos FC, et al. (2017), na qual se discutiu uma expressiva quantidade de estudos acerca dessa temática, mas não se identificou a produção de softwares que apoiem o PE em níveis secundários de atenção à saúde; em um único trabalho foi descrita a utilização de sistemas em Unidade Básica de Saúde (UBS); e dos 15 estudos analisados apenas três contemplaram todos os setores de um hospital.

Considerando a quantidade incipiente de softwares e a existência daqueles que privilegiam o cuidado em certos níveis de atenção à saúde, tais elementos constituem-se como fatores limitantes ao avanço de estratégias que ofereçam um cuidado de enfermagem mais eficiente, integral e especializado, uma vez que, para Brasil (2016), a organização da rede de serviços para o cuidado dos pés de pessoas com DM deve levar em conta, entre outros indicadores, a disponibilidade de equipamentos para o exame físico e para realização de procedimentos, como por exemplo, softwares para documentação e apoio a prática de enfermagem, com fluxos partindo idealmente sempre da Atenção Básica (AB).

\section{CONSIDERAÇÕES FINAIS}

O estudo de revisão permitiu identificar a inexistência de artigos sobre a elaboração de softwares voltados ao cuidado de pacientes portadores de UD guiados pelo PE. Nos estudos analisados, a enfermagem vem adequando seu saber científico aos avanços tecnológicos em diversos contextos assistenciais. Há diversos benefícios advindos disso, e ainda há fragilidades a serem superadas. Para isso faz-se necessário o engajamento dos cursos de graduação em enfermagem, das instituições de saúde e de fomento a pesquisas, na elaboração de estratégias de incentivo a atividades de ensino, pesquisa e extensão que evidenciem a importância da inserção tecnológica ao apoio à tomada de decisão dos enfermeiros na assistência a indivíduos portadores de UD. Quanto as ferramentas elaboradas para o atendimento a pacientes sob a égide do PE, poucas contemplavam a totalidade das etapas do método, como exemplo a inexistência da determinação de metas e resultados de enfermagem, deixando de exigir o cuidado de maneira integral. Dessa forma, considerando a multidimensionalidade do cuidado aos pacientes portadores de UD, e a complexidade do quadro clínico desses pacientes, a presente revisão tornou evidente a necessidade de incentivos para construção de softwares voltados para o cuidado a essa população, que atendam aos requisitos de flexibilidade, dinamicidade e que sejam clinicamente abrangentes, sem perder 0 olhar holístico tão primado pela enfermagem.

\section{REFERÊNCIAS}

1. APELQVIST J, et al. International consensus and practical guidelines on the management and the prevention of the diabetic foot. Diabetes Metab Res Rev, 2000; 16(1): 84-92. 
2. BARBOSA RFM, et al. Incidência de neutropenia induzida por quimioterapia no tratamento do câncer colorretal. Rev Rene, 2019; 20: e33884.

3. MINISTÉRIO DA SAÚDE (BR). Manual do pé diabético: estratégias para o cuidado da pessoa com doença crônica. Brasília (DF); 2016.

4. CAMARGO FC. Produção nacional sobre Softwares apoiadores da atuação de enfermeiros hospitalares. J Health Inform, 2018; 10(4): 125-130.

5. CARVALHO EC, et al. Obstáculos para a implantação do Processo de Enfermagem no Brasil. J Nurs UFPE on line, 2007; 1(1): 95-99.

6. CONSELHO FEDERAL DE ENFERMAGEM. Resolução №. 358, de 15 de outubro de 2009. Dispõe sobre a sistematização da assistência de enfermagem e a implementação do processo de enfermagem em ambientes públicos e privados em que ocorre o cuidado profissional de enfermagem e dá outras providências. Brasília: COFEN; 2009.

7. DAL SASSO GTM, et al. Processo de enfermagem informatizado: metodologia para associação da avaliação clínica, diagnósticos, intervenções e resultados. Rev Esc Enferm USP, 2013; 47(1): 242-249.

8. DOMINGOS CS, et al. The application of computerized nursing process: integrative review. Enferm Glob, 2017; 16(48): 603-652.

9. GARCIA TR, NÓBREGA MML. Nursing Process: from theory to the practice of care and research. Esc Anna Nery, 2009; 13(1): 188-193.

10. INTERNATIONAL DIABETES FEDERATION-IDF. Diabetes Atlas Sixth Edition. Bruxelas: International Diabetes Federation; 2013.

11. JUNIOR MGS, et al. Software para Sistematização da Assistência de Enfermagem em unidade de internação hospitalar. Rev Bras Enferm, 2018; 71(5): 2425-2431.

12. LIMA JJ, et al. Computerized nursing process: development of a mobile technology for use with neonates. Rev Bras Enferm, 2018; 71(3): 1273-1280.

13. LUCOVEIS MLS, et al. Degree of risk for foot ulcer due to diabetes: nursing assessment. Rev Bras Enferm, 2018; 71(6): 3041-3047.

14. MELNYK BM, FINEOUT-OVERHOLT E. Making the case for evidence-based practice. In: Melnyk BM, FineoutOverholt E. Evidence based practice in nursing \& healthcare. A guide to best practice. Philadelphia: Lippincot Williams \& Wilkins; 2005; 3-24

15. MELO ECA, et al. Plataforma PEnsinar $\AA$ : ferramenta de aprendizagem para o ensino do processo de enfermagem. Rev Bras Enferm, 2018; 71(4): 1522-1530.

16. MIRANDA LN, et al. Decision-making system for nursing: integrative review. Rev Enferm UFPE online, 2017; 11(10): 4263-4272.

17. PAESE F, et al. Structuring methodology of the computerized nursing process in emergency care units. Rev Bras Enferm, 2018; 71(3): 1079-1084.

18. PISSAIA LF, et al. Impacto de tecnologias na implementação da sistematização da assistência de enfermagem hospitalar: uma revisão integrativa. R Epidemiol Control Infec, 2018; 8(1): 92-100.

19. PRESSMAN RS, MAXIM BR. Engenharia de software: uma abordagem profissional. Amgh Editora; 2016.

20. SANTOS SR, NOBREGA MM. A busca da integração teoria e prática no sistema de informação em enfermagem. Enfoque na teoria fundamentada nos dados. Rev Latino-am Enfermagem, 2004; 12(3): 460-468.

21. SILVA CL, CAVALHEIRO MA. Sistema de apoio ao diagnóstico de enfermagem: uma necessidade no trabalho do enfermeiro. Ciênc Biol Saúde, 2012; 17(2): 109-112.

22. SILVA IAS, et al. O ensino do processo de enfermagem. Rev enferm UFPE online, 2018; 12(9): 2470-2478.

23. SILVA KL, et al. Desenvolvimento de software para apoiar a tomada de decisão na seleção de diagnósticos e intervenções de enfermagem para crianças e adolescentes. Rev Latino-Am Enfermagem, 2015; 23(5): $927-935$.

24. SOARES CB. Integrative review: concepts and methods used in nursing. Rev Esc Enferm USP, 2014; 48(2): 335345.

25. SOUZA NR, et al. Fatores predisponentes para o desenvolvimento da lesão por pressão em pacientes idosos: uma revisão integrativa. Estima, 2017; 15(4): 229-239. 